occurring at a longer time of 51-90 seconds. Even more interesting, there was a small secondary peak at $111+$ seconds. Analysis of the TTT in other subjects with various diseases suggests that the existence of a long TTT may have some clinical significance. A highly significant correlation between plasma and leucocyte AA values was found and a significant relationship between TTT and leucocyte values. There was, however, no relationship between plasma AA and TTT. It can therefore be concluded that TTT, like leucocyte AA values, provides a measure of tissue AA storage and metabolic requirements.

Serum cholesterol levels were estimated in the G.S. group as part of a screening programme for coronary heart disease conducted by the Irish Heart Foundation. In the younger age groups there was no significant correlation between serum cholesterol and TTT. There was, however, a significant correlation between cholesterol and tissue AA values in the tongue in the oldest age group.

The tongue test provides a simple and quick method for measuring the turnover rate and metabolism of AA in the tongue and is representative of the tissue status of AA. The AA tongue test time, like that of the AA concentration in the leucocytes, falls with increasing age. It shows certain population characteristics which may be associated with pathological conditions.

\section{Screening for Coronary Heart Disease Risk Factors.} N. HickeY (Irish Heart Foundation).

In 1969 the Irish Heart Foundation began a screening programme for coronary risk factors. The object of the study is to test the acceptability of such a programme and to determine the yield of high-risk individuals.

The screening procedure was outlined and the risk categories of the initial 10,000 male subjects presented. The prevalence of overt coronary heart disease and of high-risk category individuals was approximately $19 \%$ of those screened.

A follow-up study among the general population and in industry will shortly start to determine the extent to which the risk factor status of individuals can be altered.

\section{Second Session (Chairman: J. Pemberton)}

The Association of Place of Birth in South Wales with Central Nervous System Malformation Prevalence. C. J ROBERTS (Dept. of Social and Occupational Medicine, Welsh National School of Medicine, Cardiff).

There is good evidence that enviromental influences play some part, possibly a major part, in the aetiology of neural-tube malformation in the human embryo. A strong pointer in this direction is the degree to which malformations vary from country to country and from area to area within countries.

For three years beginning 1 January 1964 information about all infants born to women resident in a defined area in South Wales and about all the congenital defects identified in the birth population has been collected. The area surveyed was defined by the county boundaries of Glamorgan and Monmouthshire. In the three years of the investigation, 92,980 infants (live and still births) were born to women resident in these areas; of these infants 90,921 were the outcome of singleton pregnancies and 740 of these were ascertained to have neural-tube defects:

Marked differences in area prevalence were observed particularly high rates were recorded in certain mining ${ }^{+}$ valleys of Monmouthshire and those of East and Wese Glamorgan. When (for the purposes of more detaile $\bar{F}$ statistical analysis) the total sample was divided into eigh subpopulations defined (before data were collected) oा the basis of certain geographical, occupational, and cultural characteristics, area differences (although not so prominent) were still in evidence. These ranged from $9 \cdot \vec{\phi}$ per 1,000 singleton births in the eastern mining valleys of Glamorganshire to 5.3 for Newport County Boroughw However, since parity, social class, and maternal age were also found to be strongly associated with CNS mafe formation prevalence, the problem was to determine the extent to which the observed association between areas and malformation prevalence was dependent upon thesecondary association of both variables with parity social class, and maternal age. This was approached by use of multivariate analysis in which the dependent and independent variables were measured on a binary scale.

The results of this analysis showed that adjustment for biological parity, social class, and maternal age furthefD reduced the magnitude of the area differences reportec above. On the basis of these findings it is concluded tha巴 in South Wales the effect of area (defined in this study on the basis of occupation-mining/non-mining fido geographical—east/west, valleys/coastal plan criteria)ißv considerably less than that of biological parity, proba角 $\dot{y}$ less than that of social class, but greater than that 0 maternal age.

Social Attitudes to and Utilization of Cervical Cance Tests. N. D. RICHARDs and P. J. M. McEwan (Centre for Social Research, University of Sussex, Brighton).

The Yield of Disease obtained at Screening a Middle Aged Population. Harriet Trevelyan (Dept. of Clinicat Epidemiology, St. Thomas's Hospital Medical School London).

The South East London screening study is a controlled trial of multiphasic screening and surveillance in middle age. The aim is to evaluate the effect of such screening i terms of (1) the long-term effect on morbidity and moro tality, and (2) the yield of previously unknown disease which is considered to require management.

The study population includes all individuals between 40 and 64 years in nine general practitioners' lists in tw group practices. This population is randomly allocated to. screening and control groups. The screening group is invited for screening every two years. The screening process consists of a symptom questionnaire, anthro pometry, electrocardiography, respirometry, blood presw sure recording, tests of vision and hearing, blood tests? and a physician's examination.

Of those allocated to the screening group, 3,100 in dividuals were known to be still on the practice list Of these $73 \%$ accepted and were screened. The refusers were older, had a lower consultation rate in the previous 
year, and were concentrated in certain general practitioners' lists.

There was a yield of $\mathbf{1} \cdot \mathbf{2}$ conditions per person screened; $47 \%$ of the conditions were previously known to the medical service. Of all conditions found, $56 \%$ were classified as minor and only $10 \%$ as major or life threatening.

These results show that only a small proportion of conditions found were considered to be life threatening. The majority of these major conditions were already known to the medical service; $15 \%$ of those previously unknown required observations, drugs or operation. In contrast, one-half of the minor conditions were previously unknown and $10 \%$ required management.

Evaluation of Screening Tests for Unreported Disability in the Elderly. JOCELYN CHAMBERLAIN (London School of Hygiene and Tropical Medicine).

Many studies of elderly people have shown a large prevalence of medical and social disability much of which, although it can probably be alleviated, is not known to the health and social services. Screening as a means of bringing this unreported need to light is frequently recommended, but so far there has been little evaluation of practical methods of testing for these disorders.

This paper described a study which aims to measure the error rates and repeatability of screening questions and tests used by nurses to screen elderly people at home for four common disabilities-impairments of vision, of hearing, and of mobility, and financial need. To determine the validity of screening in this way, it is necessary for each subject to be seen three times, being screened twice and examined definitively once.

People over the age of 70 on the list of a group practice are being screened in a domiciliary interview conducted by a nurse. A few days later each subject is visited again and the screening procedure is repeated; by comparison of the results from the two interviews, the repeatability of the screening questions and tests can be calculated. Subsequently each person is given a specialist definitive examination at a clinic of each of the systems being studied. By comparing the findings of this with the findings of screening, the false negative rate, false positive rate, and predictive value of each screening test can be worked out.

Results from the pilot survey indicate that screening is satisfactory for visual, auditory, and foot disabilities, and improvements have been made in screening for general impairment of mobility and for financial need. It is hoped that when the main survey is completed a reasonably valid instrument for screening elderly people for all these disabilities will have been developed.

\section{ThIRD SESSION (Chairman: M. D. WARREN)}

The Distribution of General Practitioners in England. J. R. Butler, J. M. BeVAN and R. C. TAYLOR (University of Kent at Canterbury).

The study investigated patterns of mobility and settlement among general practitioners in England and examined the effect of the Designated Areas Allowance, first introduced in 1966, on the distribution of family doctors. The data are drawn mainly from a postal survey conducted in 1969/70 among a 1 in 8 sample of all principals in designated areas and a 1 in 10 sample of principals in non-designated areas. The response rate was $85 \%$; the total number in the survey was 1,721 .

The majority of respondents had changed practices at least once during their careers as GPs, often moving across county and regional boundaries in the process. Only $40 \%$ had remained in the same practice up to the time of the survey, and as many as one-third had moved at least once across standard regional boundaries. The influence of birthplace, family home area, and medical school was examined, and it was shown not only that a doctor's chances of returning to an area increase with the number of ties he has, but also that the influence of each was interdependent with the others.

The assumption that the designated areas are professionally deprived and socially depressed was found wanting. A series of questions about various social and professional aspects of life in each type of area yielded no substantial distinguishing features: in some cases the designated doctors enjoyed superior facilities, in other cases it was doctors in non-designated areas who recorded higher scores of satisfaction. It is estimated that the Designated Areas Allowance was paid to about 800 principals in England in 1968 with personal lists below 2,500 , and automatically withheld from about 5,000 principals with lists above this size who were in nondesignated areas.

The Role and Function of the Practice Nurse from the Patient's Point of View. Diane J. Cunningham, J. M. BEVAN and G. B. FloYD (University of Kent at Canterbury).

This paper reported on the one aspect of a study designed to evaluate a number of innovations in a group practice in a London borough, viz., the assessment of patients' attitudes to the role of the nurse in general practice. It was hypothesized that a number of variables might influence the attitudes of patients to the practice nurse; these were sex, age, marital status, social class, education, frequency of interaction with the doctor, the nature of the doctor-patient relationship, and previous contact with the nurse. Information was obtained from two sources-a postal survey of a 1 in 8 random sample of the entire practice population in the age range 18-64, complemented by an interview inquiry of a separate 1 in 6 random sample from one of the practice doctor's list. Both surveys produced a high response rate (postal survey $73 \%$; interview inquiry $81 \%$ ). The results from the two surveys were similar.

Over three-quarters of all the respondents felt that the nurse was an important aid to the doctor, whereas only half felt that the nurse was an advantage to the patient. Respondents who had experienced the nurse assisting the doctor reacted more favourably towards the practice nurse than those who had not, independently of sex, age, marital status, education, or frequency of visits to the doctor. Broadly speaking the manual classes expressed the most consistently favourable opinions towards the 